\title{
Near-conformal window assembly for airborne payloads: improved time on- station and optical performance
}

Hinrichs, Keith, Roll, Christopher, Berkson, Joel, Sebastian, Thomas

Keith M. Hinrichs, Christopher D. Roll, Joel D. Berkson, Thomas Sebastian, "Near-conformal window assembly for airborne payloads: improved time onstation and optical performance," Proc. SPIE 11175, Optifab 2019, 1117512 (15 November 2019); doi: 10.1117/12.2537076

SPIE. Event: SPIE Optifab, 2019, Rochester, New York, United States 


\title{
Near-conformal window assembly for airborne payloads: improved time on-station and optical performance
}

\author{
Keith M. Hinrichs ${ }^{\mathrm{a} 1}$, Christopher D. Roll ${ }^{\mathrm{a}}$, Joel D. Berkson ${ }^{\mathrm{b}}$, Thomas Sebastian ${ }^{\mathrm{a}}$ \\ ${ }^{a}$ MIT Lincoln Laboratory, 244 Wood St, Lexington MA, 02421 USA; ' University of Arizona, \\ Optical Sciences Center, Tucson AZ, 85721 USA
}

\begin{abstract}
Conventional windows for airborne payloads are often discontinuous with the aircraft or pod skin. A protruding structure or hollow cavity increases aerodynamic drag, which consumes more fuel and thus reduces the amount of time available on-station. These geometries give rise to turbulent aero-optical effects, which can reduce the payload's optical performance because it has to see through turbulence.

This paper describes a multi-paned or segmented window concept that matches the local topology of the aircraft pod or skin. This approach is suitable for optical payloads having multiple fixed fields-of-view such as staring infrared search and track systems, but not scanning systems. This approach for creating a near-conformal window assembly should be particularly useful for rapid prototyping of windows for airborne optical payloads, providing a nearer-term alternative to monolithic windows that are ground and polished into complex shapes.

In this paper, a 14-inch diameter pod faring with three window segments was chosen as a point design for a notional airborne optical payload. Fused silica planar windowpanes were fabricated with matching, mating mitered edges. The panes were chemically bonded directly to each other with a sodium-silicate solution. The bonding process and fixturing are described. The resulting glass bond is strong and minimizes the non-useable seam between panes. This approach increases the clear aperture of each pane compared with windowpanes bonded into individual mechanical bezels. Interferometric measurements of the prototype show no degradation in transmitted wavefront error after silicate bonding.
\end{abstract}

Keywords: airborne, aero-optical, conformal, IRST, payload, segment, silicate bond, turbulence, window, windowpane

\section{OVERVIEW}

\subsection{Problem statement}

Conventional windows for airborne optical payloads are often discontinuous with the aircraft or pod skin (known as the outer mold line, or OML). A protruding structure, as shown in Figure 1, or a hollow cavity increases aerodynamic drag, which consumes more fuel and thus reduces the amount of time available on-station for ISR collection. These aerogeometries also gives rise to turbulent aero-optical effects, which can reduce the optical performance of the payload as it must see through the turbulence.

\footnotetext{
${ }^{1}$ keith.hinrichs@ll.mit.edu; www.II.mit.edu
}

Optifab 2019, edited by Blair L. Unger, Jessica DeGroote Nelson, Proc. of SPIE Vol. 11175, $1117512 \cdot$ (c) $2019 \mathrm{SPIE} \cdot \mathrm{CCC}$ code: $0277-786 \mathrm{X} / 19 / \$ 21 \cdot$ doi: $10.1117 / 12.2537076$ 




Figure 1. Gimbal on underside of unmanned aerial vehicle (UAV) ${ }^{1}$

\subsection{Concept}

The work presented here seeks to improve the window assemblies used in optical payloads that have multiple fixed fieldsof-view. Applications that would benefit from near-conformal segmented window assemblies include multiplexed staring infrared search and track systems (IRST) ${ }^{2}$, hyperspectral sensors with multiple fixed fields-of-view, and LADAR systems. This approach may be useful for some scanning systems if mitigation is provided for the reduced optical performance caused by a divided aperture and/or differential wedge ${ }^{3,4,5}$.

A multi-paned or segmented window assembly is designed to match the local topology of the aircraft or pod skin ${ }^{6}$. The planar windowpanes are fabricated with matching, mating mitered edges ${ }^{7}$. The panes are bonded directly to each other ${ }^{8}$ (i.e., no interstitial frame) using a chemical bond or structural adhesive, as shown in Figure 2. For this effort, fused silica glass panes were chemically bonded to each other with a sodium-silicate (liquid glass) solution. This is called "silicate bonding" in the rest of this paper. The resulting glass bond is strong and minimizes the non-useable seam between panes. This approach increases the clear aperture (useable portion) of each pane, compared with windowpanes bonded into individual mechanical bezels.

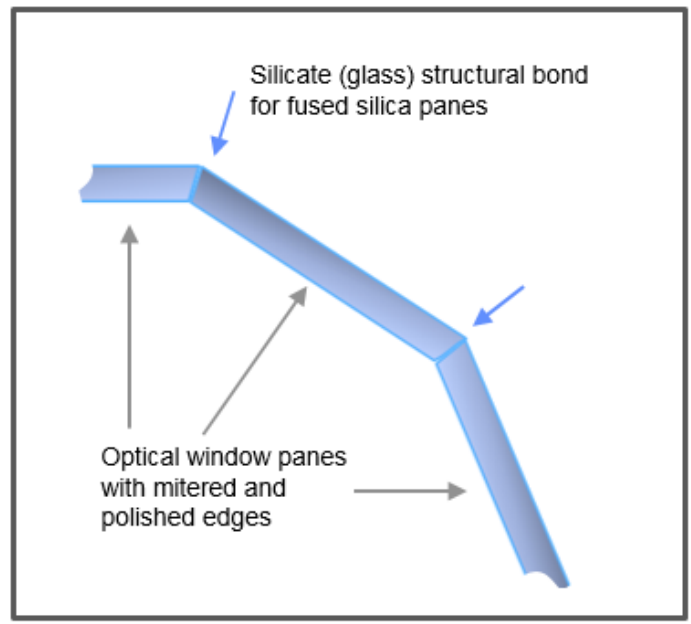

Figure 2. Near-conformal window assembly technical approach

The proposed window concept is nearly conformal to the aircraft's OML, reducing the turbulence at the outside window boundary and thereby improving the aero-optical performance of the sensor (e.g., PSF, MTF, Strehl, SNR, and/or pointing jitter $)^{3,4,6}$. Reduced turbulence also reduces the aerodynamic drag which decreases fuel consumption, allowing the aircraft to spend more time-on-station. ${ }^{9,10}$ 
This segmented pane approach for creating a near-conformal window assembly offers a lower cost and shorter lead-time alternative to monolithic curved windows that are ground and polished into toroidal, ogive, freeform, hyperbolic, or other complex shapes. At the least, it provides a geometric approximation for fast prototyping while the monolithic, complexshaped production window is being fabricated ${ }^{9}$.

Industry has utilized this approach for optical payloads such as the F-35's electro-optical targeting system (EOTS) 7 , 11, the wing pylon-mounted Sniper advanced targeting pod (ATP) $)^{12}$, both shown in Figure 3, and the Avionics Flying Laboratory (AFL) testbed ${ }^{3}$.



Figure 3. Industry examples of near-conformal windows on F-35's EOTS ${ }^{11}$ and the Sniper ATP ${ }^{12}$

\subsection{Technical approach}

The goal of this effort was to establish an assembly process for building prototype near-conformal windows for an aircraft or pod-mounted optical payload that has multiple field-of-views. The following approach was used:

1. Create a point-design for a near-conformal window sub-assembly that could be used with a pod-mounted payload. Use fused silica windowpanes with matching mitered edges that will be silicate-bonded to form the near-conformal shape of the window sub-assembly.

2. Design a pod fairing to mount the window assembly into to demonstrate the capability for future programs.

2. Analyze expected aerodynamic performance.

3. Develop the bonding process needed to fabricate this prototype. 
4. Procure windowpanes, frame, fairing, and fixturing. Silicate-bond the panes together along their polished edges.

5. Measure transmitted wavefront error as a function of clear aperture to determine any degradation due to the bonding process.

6. Bond the three-pane window assembly into the frame with compliant aerospace adhesive and attach a notional fairing.

\section{PROCESS DEVELOPMENT WITH CO-PLANAR WINDOW COUPONS}

Silicate bonding (hydroxide catalysis) uses a mixture of sodium-silicate solution (Sigma-Aldrich 338443) and deionized water (1:6 by volume) to form a covalent chemical bond between fused silica pieces ${ }^{13}$. The resulting bond line is a fraction of a micron thick. Silicate bonds are known for their high mechanical strength and stability. Fused silica parts or silicon dioxide coated metals can be bonded together with this approach. The solution crystallizes between the mating surfaces and forms a glass bond, which cures at room temperature. The water content can be driven out more quickly when the subassembly is held at an elevated temperature of $90^{\circ} \mathrm{C}$.

Test coupons for co-planar bonding were procured from Edmund Optics (part number 48-201). The parts are 1 x 1 x 0.125 inches with fine ground, $90^{\circ}$ mitered edges. The Massachusetts Institute of Technology Lincoln Laboratory's group 82 Optics Shop polished two edges on opposing sides to $\lambda / 4 \mathrm{p}$-v flatness. The edges were cleaned by drag-wiping with acetone then methanol prior to bonding. Figure 4 describes the silicate-bonding process used with these coupons, which is the same process used for bonding the subsequent prototype window sub-assembly.

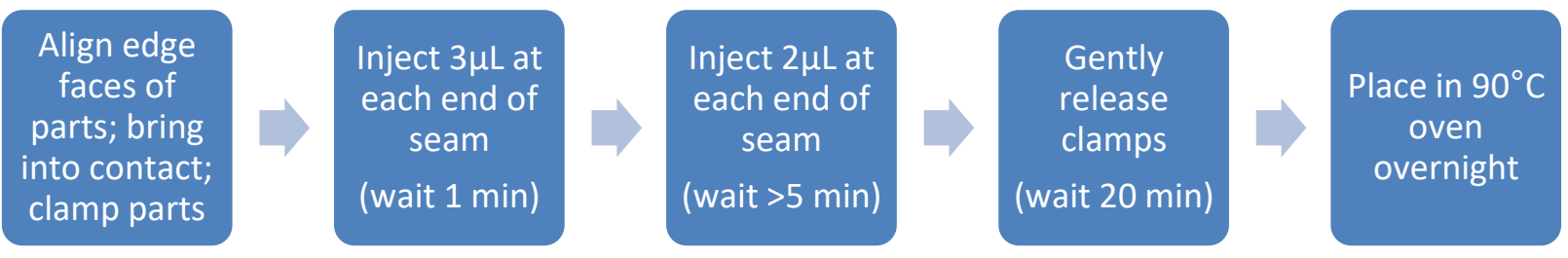

Figure 4. Silicate bonding process

The bonded co-planar window sub-assembly is shown in Figure 5. These inexpensive windows allowed for a rapid learning curve with respect to mounting, aligning, solution dispensing, curing, and handling of silicate-bonded sub-assemblies.

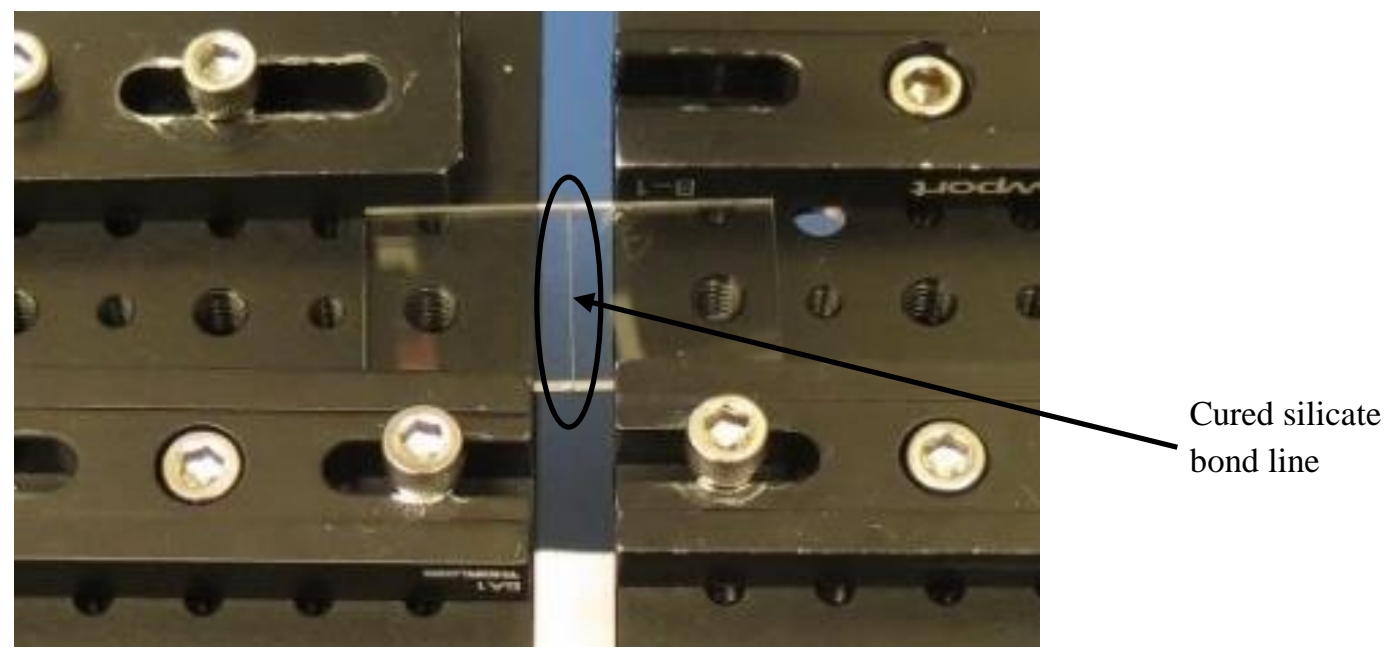

Figure 5. Bonded test coupons 
The individual panes were tested in transmission on a Fizeau visible interferometer before bonding. After curing, the twopane sub-assembly was tested, as shown in Figure 6.

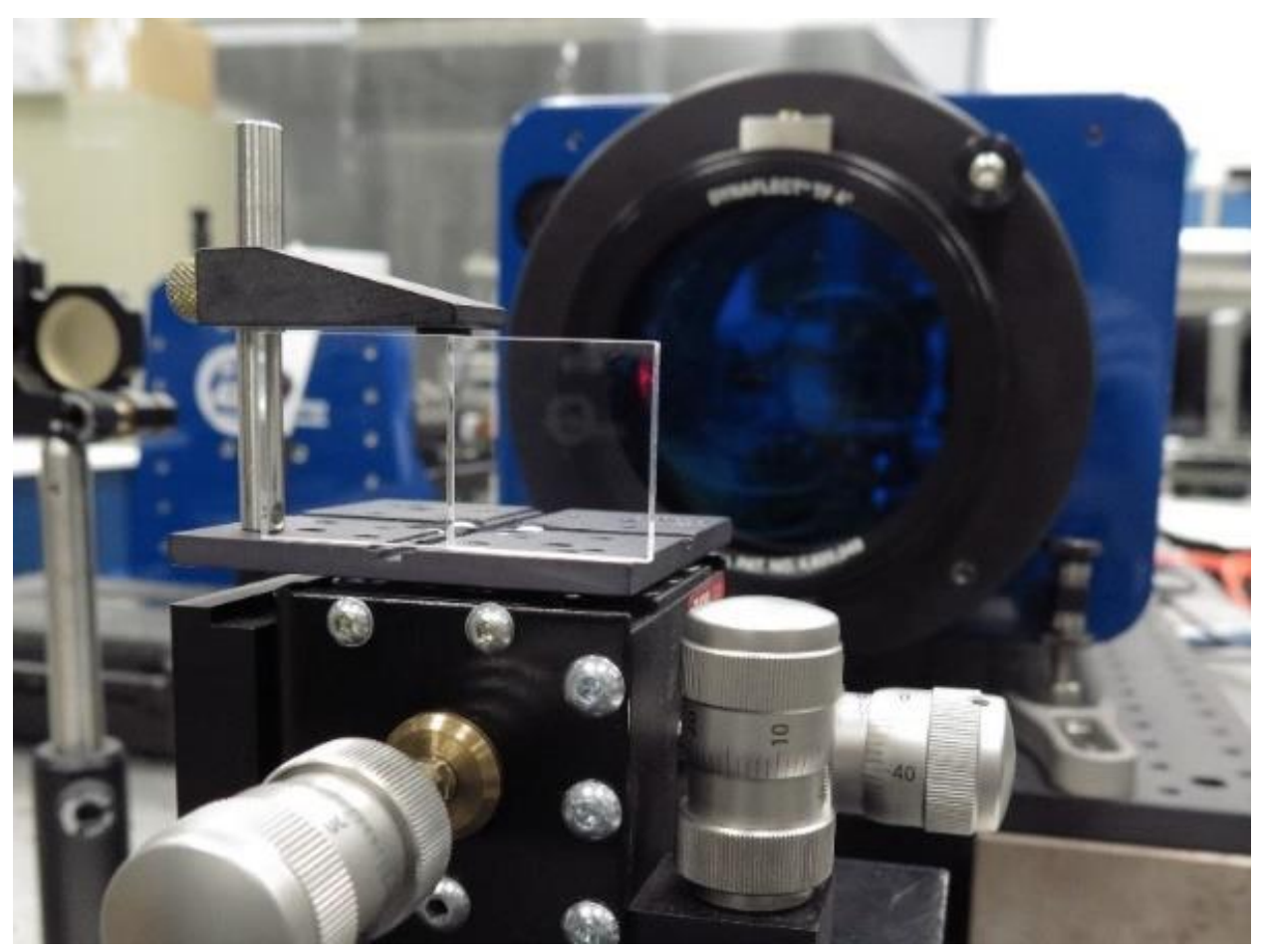

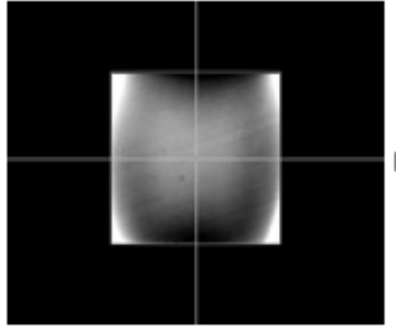

A

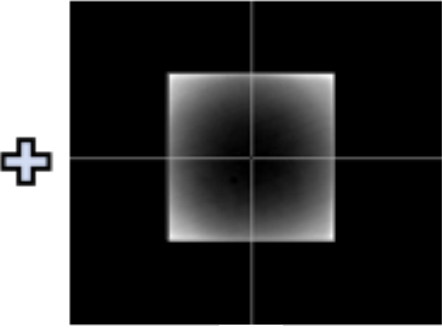

B

Figure 6. Interferometric testing of the coupons

Comparing the transmitted wavefront error of pre-bonded and post-bonded windows (see Table 1) shows that there is no effective difference in transmitted wavefront error. This indicates that there is no degradation in optical performance due to this bonding process.

Table 1. Transmitted wavefront error comparison of test coupons before and after bonding (full aperture)

\begin{tabular}{|c|c|c|}
\hline Transmitted RMS WFE & Pre-bonding & Post-bonding \\
\hline Coupon A & 0.052 waves & 0.046 waves \\
\hline Coupon B & 0.053 waves & 0.059 waves \\
\hline
\end{tabular}




\section{PROTOTYPE NEAR-CONFORMAL WINDOW ASSEMBLY}

\subsection{Design}

The prototype point design contains three windowpanes which approximate the OML of a 14 inch diameter pod. The initial half-pod concept is shown in Figure 7.

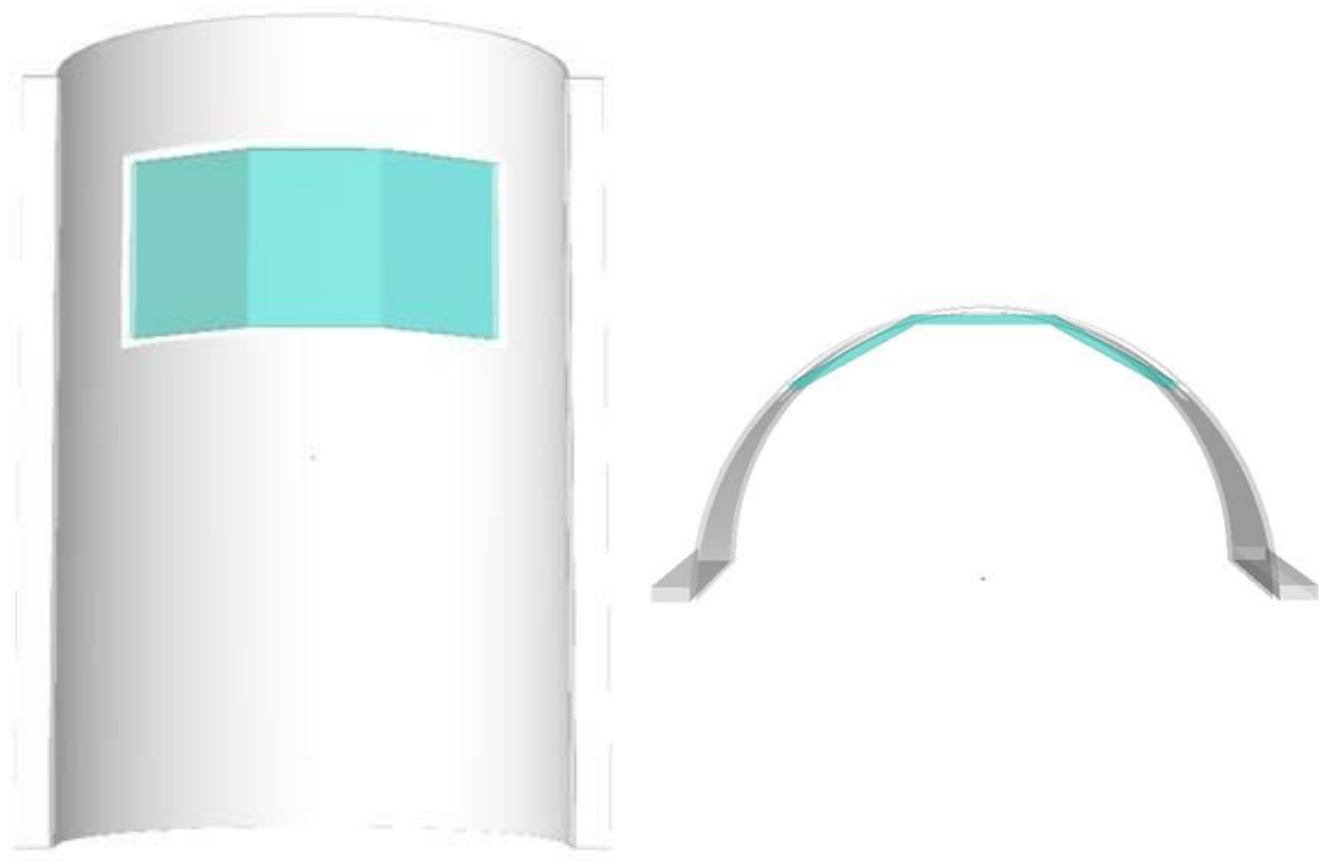

Figure 7. Initial concept for a prototype near-conformal window assembly

The fused silica panes are $3 \times 4 \times 0.30$ inches and are shown in Figure 8. This is a reasonable size-to-thickness aspect ratio for an optical window that may experience gravel impingement during takeoff and landing. The mitered angle on the long sides is $75^{\circ}$. The angled faces are polished to $\lambda / 4 \mathrm{p}$-v flatness.
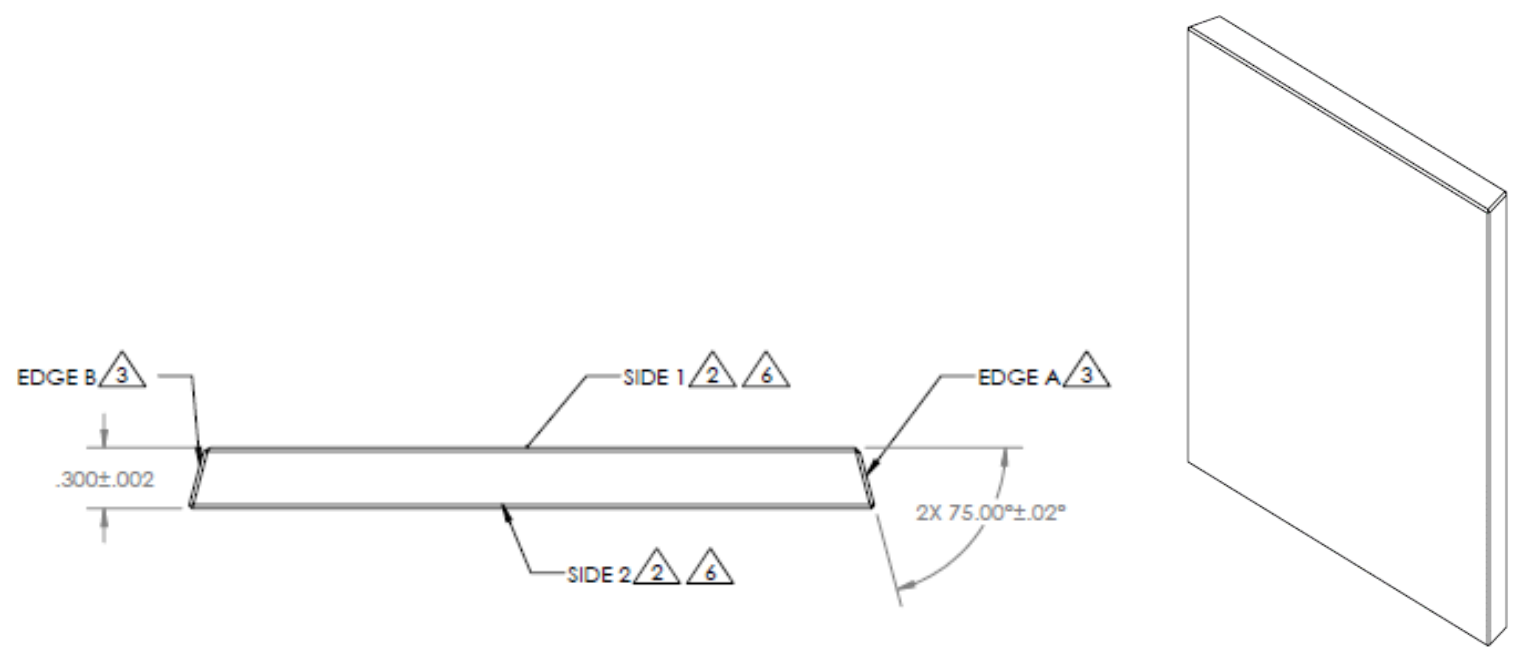

Figure 8. Prototype windowpane dimensions (inches) 
The opto-mechanical frame is machined from aluminum 6061-T6. The window sub-assembly is intended to be bonded into the frame with a mechanically-compliant aerospace-grade adhesive. The frame's brackets contain through-holes on integer-inch centers so they can be attached to an optical table during integration. An aluminum sheet was formed into a half-cylinder notional fairing and is attached to the frame with flat-head screws.

The thickness of the bond line between the window sub-assembly and the frame was optimized to provide mechanical compliance over a temperature range of -40 to $+70^{\circ} \mathrm{C}$. Initially, Momentive RTV 566 silicone adhesive was selected for its modulus over temperature, its environmental robustness, and its heritage on flight programs. However, Dow Corning 61104 silicone was ultimately selected because of its higher viscosity, making it a "non-flowing" adhesive. Additional fixtures and process development, which exceed the scope of this initiative, would have been required to dam-and-fill the bond line with RTV 566. Nevertheless, the 6-1104 silicone is an appropriate surrogate that is low outgassing and operates from $-45^{\circ} \mathrm{C}$ to $200^{\circ} \mathrm{C}$. The optimized bond line thickness is 0.125 inches along the length of the window assembly and 0.188 along the shorter edges. The final design of the prototype non-conformal window assembly is shown in Figure 9.



Figure 9. Final design of prototype near-conformal window assembly 


\subsection{Aerodynamic analysis}

The proposed design is intended to improve laminar airflow across the window to reduce turbulence. Figure 10, left, shows the aerodynamic performance of an existing pod-mounted optical payload with multiple fixed non-overlapping fields-of-view. The pod has a flat window in a recess on the bottom of the pod. Computational fluid dynamics analysis (CFD) shows that turbulence occurs in the window cavity during flight.
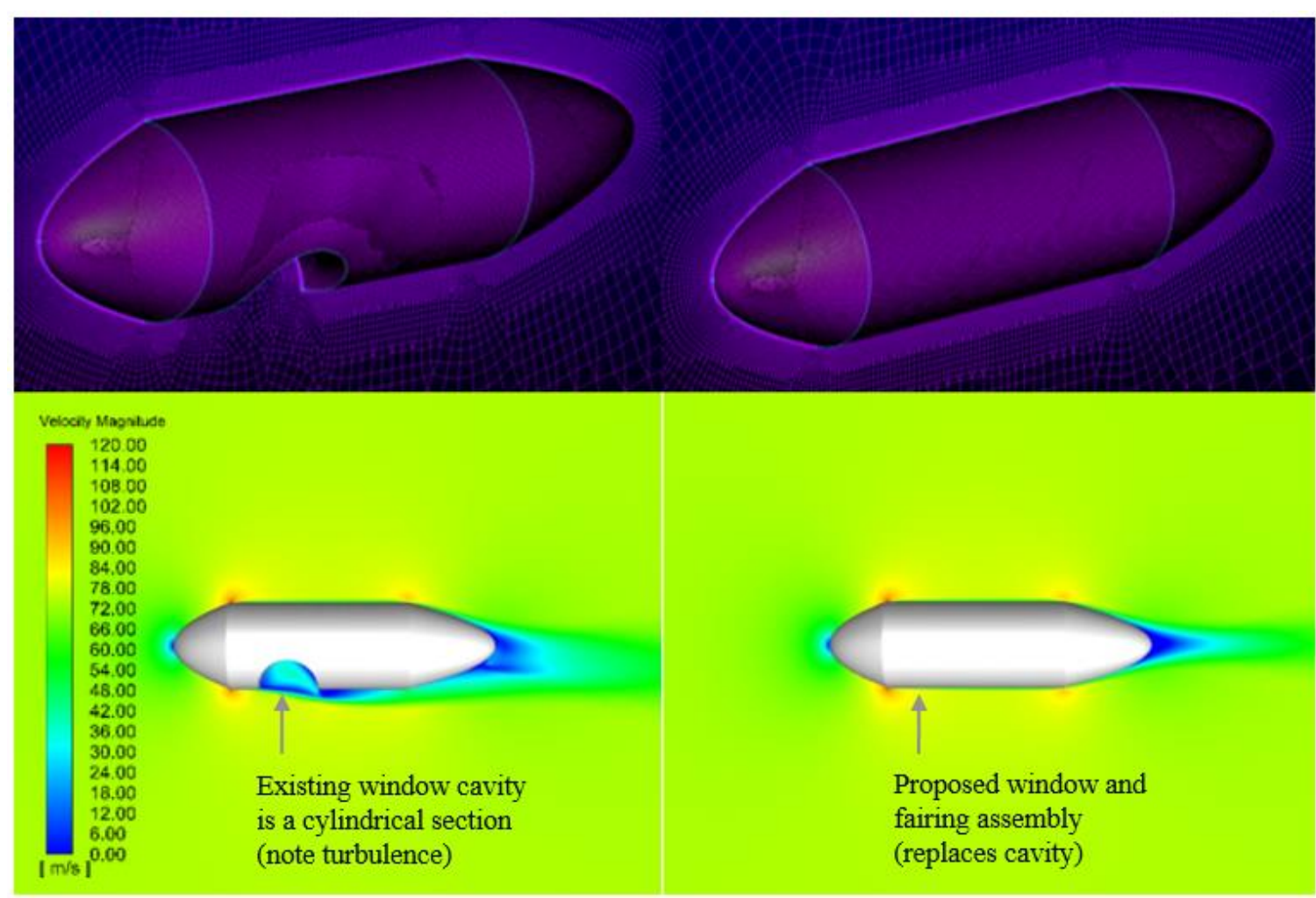

Figure 10. CFD simulation of airflow over the existing pod (left) and the proposed pod design (right)

The proposed alternative (right side of figure) removes the flat, recessed window and replaces it with a multi-pane nearconformal window sub-assembly that is located along the cylindrical pod's circumference. Each pane corresponds to one of the fixed look angles for the notional downward-looking sensor. A fairing blends the pod OML with the window subassembly, as shown in Figure 11. 




Figure 11. Proposed near-conformal window for the pod (side view and axial cross-section)

For this effort, the aerodynamic drag of the original pod and a near-conformal window alternative were analyzed using ANSYS Fluent 18.2. The pod altitude and airspeed represent what the pod would see if attached to an MQ-9 Reaper flying at 150 knots at 10,000 feet. The CFD models used a symmetric unstructured quad grid generated by Pointwise 18.0R2. The CFD models used the k-omega SST turbulence model with ideal gas assumption (compressible flow) and the 1976 US standard atmosphere model.

The analysis indicates there would be a $49 \%$ reduction in drag for this flight condition if the existing design was replaced with a near-conformal segmented window assembly. This is a significant reduction in the drag of the pod. To estimate increased time-on-station, the pod drag would be incorporated in a platform aerodynamic performance model. Decreased fuel consumption would be calculated for a given mission profile (takeoff/ferry/on-station/ferry/landing), and the fuel savings would inform the estimate for increased time on-station.

\subsection{Silicate bonding of near-conformal window sub-assembly}

A set of fixturing was first designed to hold the windowpanes during silicate bonding. The surfaces to be bonded must be co-planar and in contact with each other. Off-the-shelf opto-mechanical stages and mounting plates were purchased from Thorlabs and Newport corporations. The first two windowpanes were then mounted in the bonding fixture as shown in Figure 12. The mounts were adjusted until the surfaces to be bonded were parallel; this was verified by using plastic shim stock to create a uniform air-gap along the length of the bond line. The tilted pane was then translated until it was in direct contact with the horizontal pane's edge. 


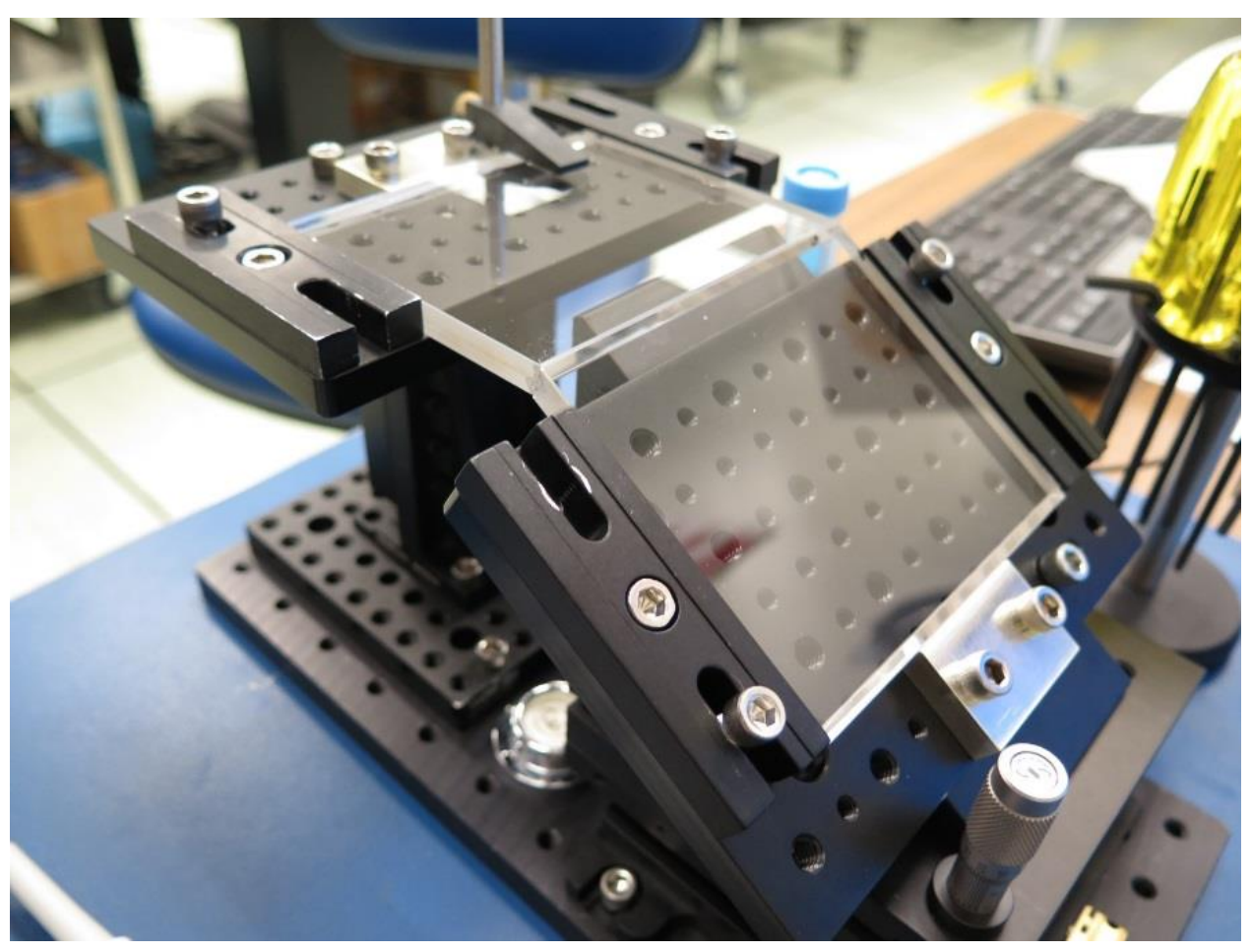

Figure 12. Two angled windowpanes in bonding fixture

The silicate solution was introduced from both ends of the joint with a micro-pipette dispenser and was allowed to wick into the bond gap until the gap was visibly filled. The solution sets up in a few minutes. The bond was allowed to cure overnight in the fixture. Then the parts were removed and placed in an oven to soak at $90^{\circ} \mathrm{C}$ for twenty-four hours. The sub-assembly was removed from the oven and allowed to return to room temperature naturally.

The two-pane set was tested on the interferometer shown in Figure 13. The test results are shown in Table 2. There was no degradation in transmitted wavefront error (TWFE) near the bond line. 


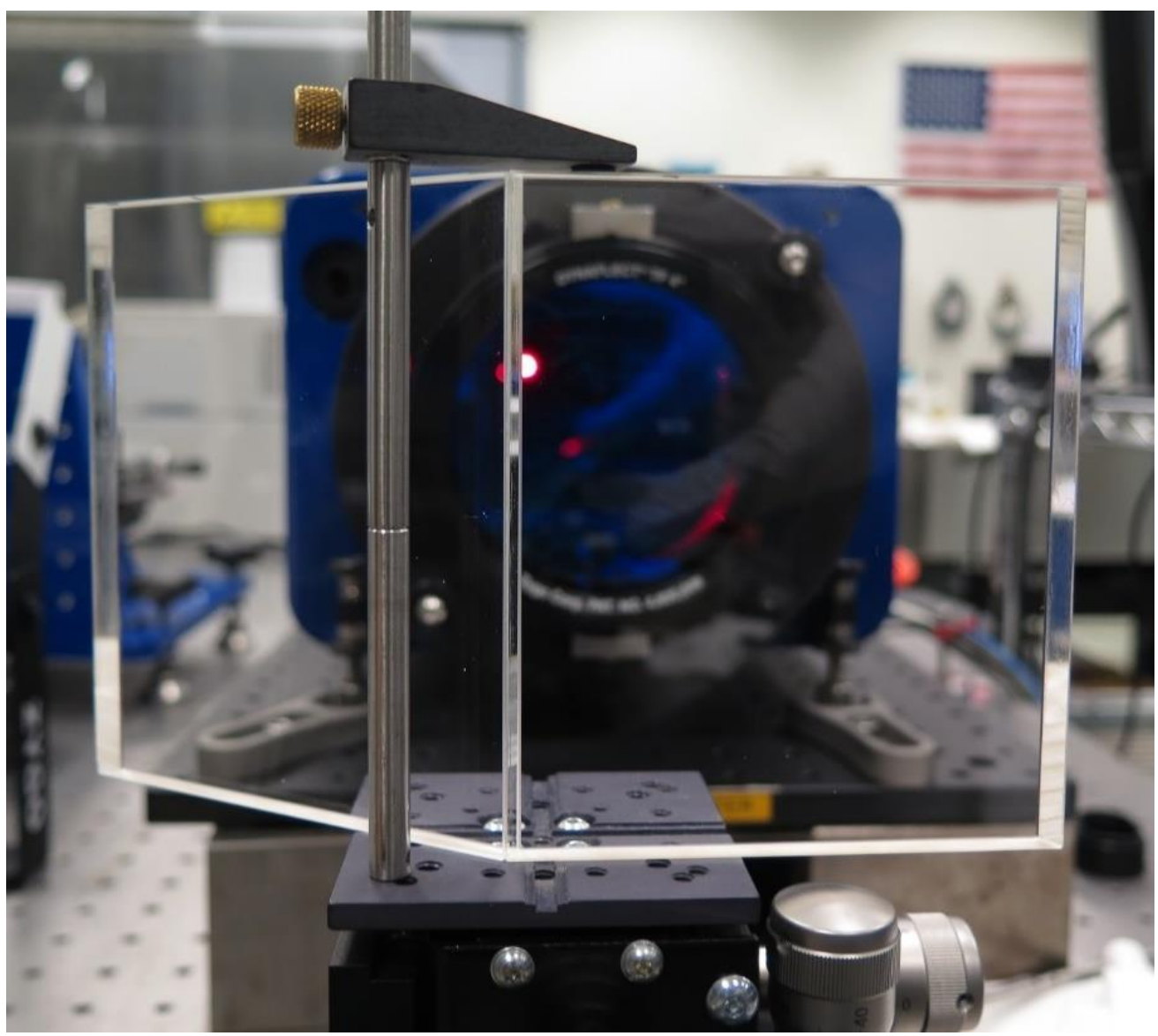

Figure 13. Two pane bonded sub-assembly

Table 2. Transmitted wavefront error comparison of two-pane angled sub-assembly

\begin{tabular}{|c|c|c|}
\hline $\begin{array}{c}\text { Transmitted RMS WFE } \\
\text { (3 inch clear aperture) }\end{array}$ & Pre-bonding & Post-bonding \\
\hline Window 1 & 0.040 waves & 0.035 waves \\
\hline Window 2 & 0.053 waves & 0.042 waves \\
\hline
\end{tabular}

The fixture was re-configured to accept the bonded pair of panes plus a third to-be-bonded pane. The third pane was positioned so that its bond surface was parallel to and in direct contact with the mating surface. See Figure 14 and Figure 15. 


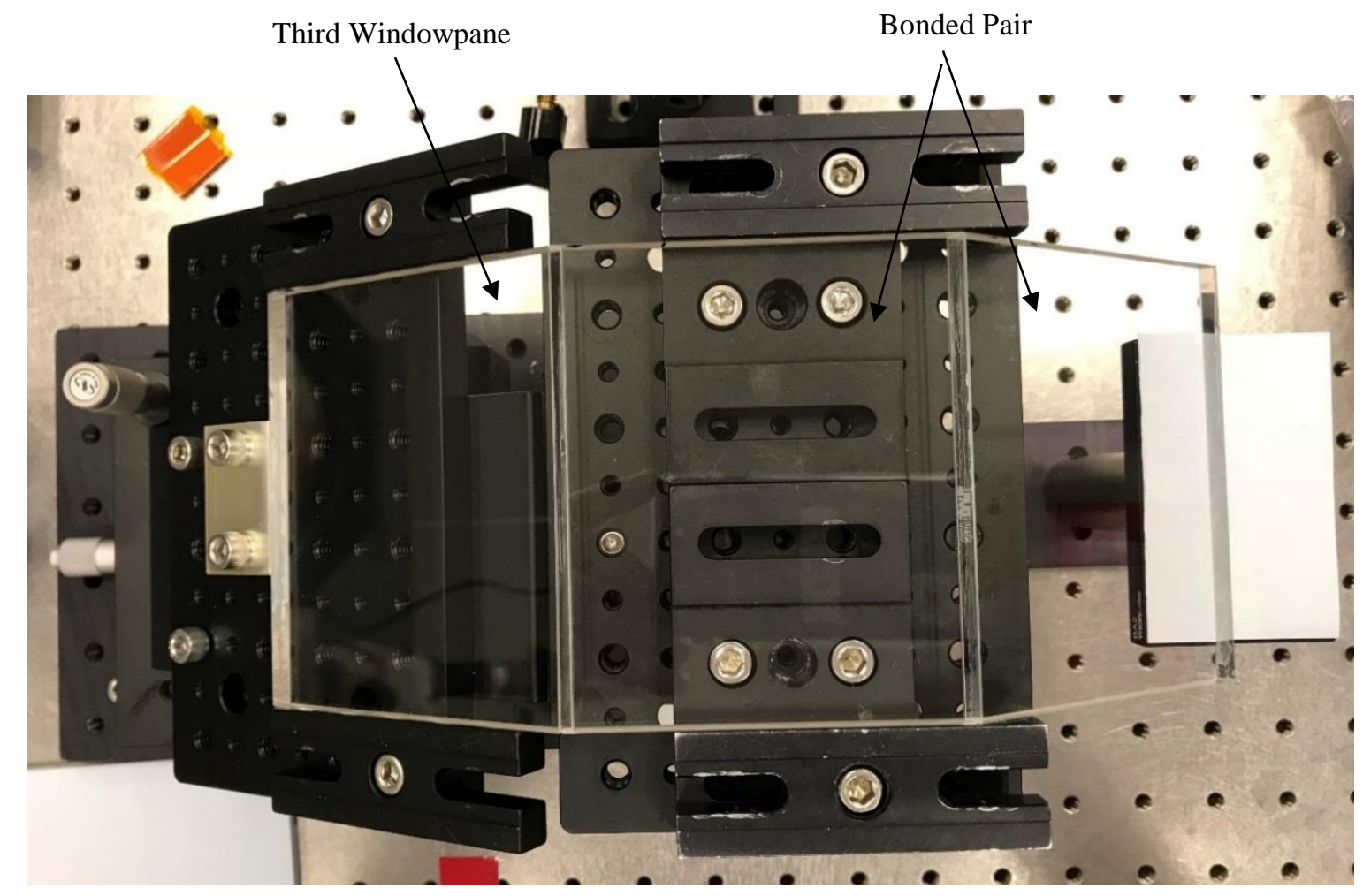

Figure 14. Fixture supporting two-pane sub-assembly and the to-be-bonded third pane



Figure 15. Interface between third and center panes

The silicate solution was injected into the bond gap in the same manner. It was allowed to cure overnight, then placed in an oven for a $90^{\circ} \mathrm{C}$ soak for twenty-four hours. It was removed and allowed to return to room temperature naturally. The bonded window sub-assembly is shown in Figure 16, with a close-up picture of the silicate bonded interfaces shown in Figure 17. 


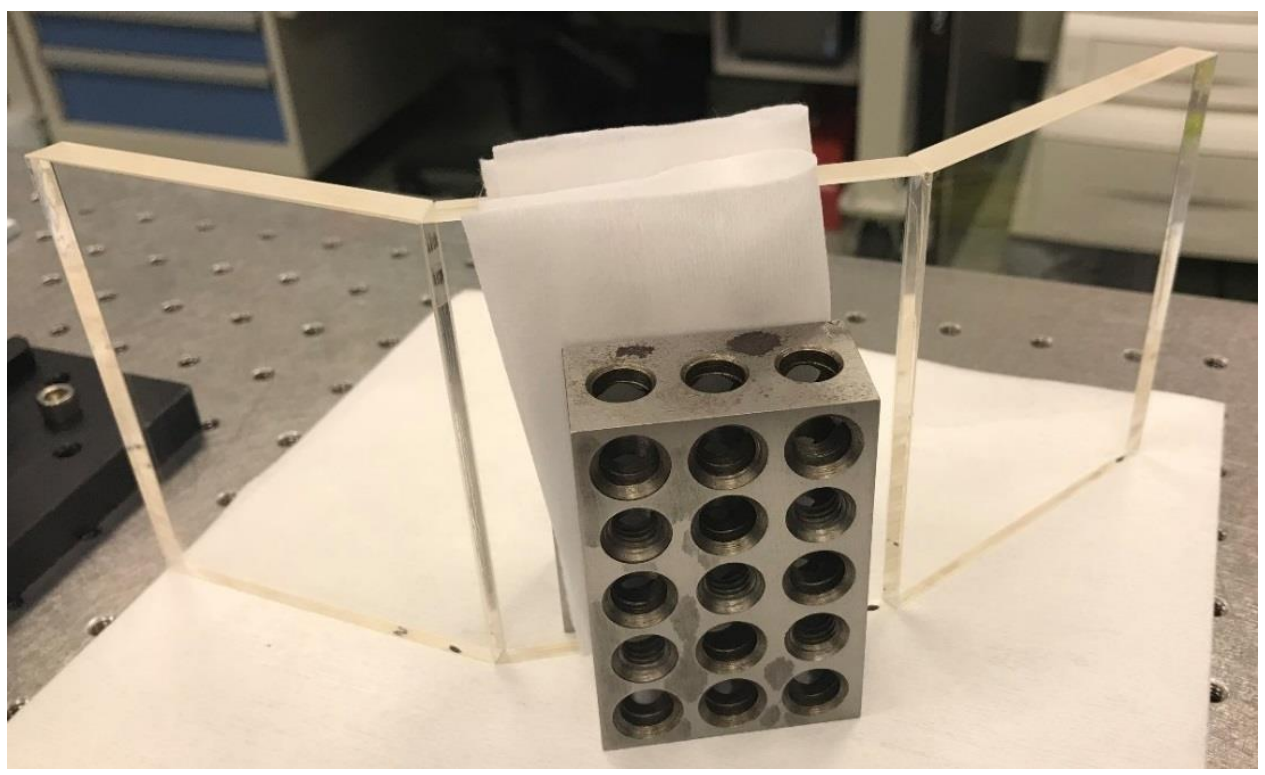

Figure 16. Bonded window sub-assembly



Figure 17. Left silicate bonded interface

\subsection{Integration of the prototype near-conformal window assembly}

The fairing was bolted onto the frame. The window sub-assembly was supported on a padded post mount and was centered within the opening of the aluminum frame with shims, as shown in Figure 18. 


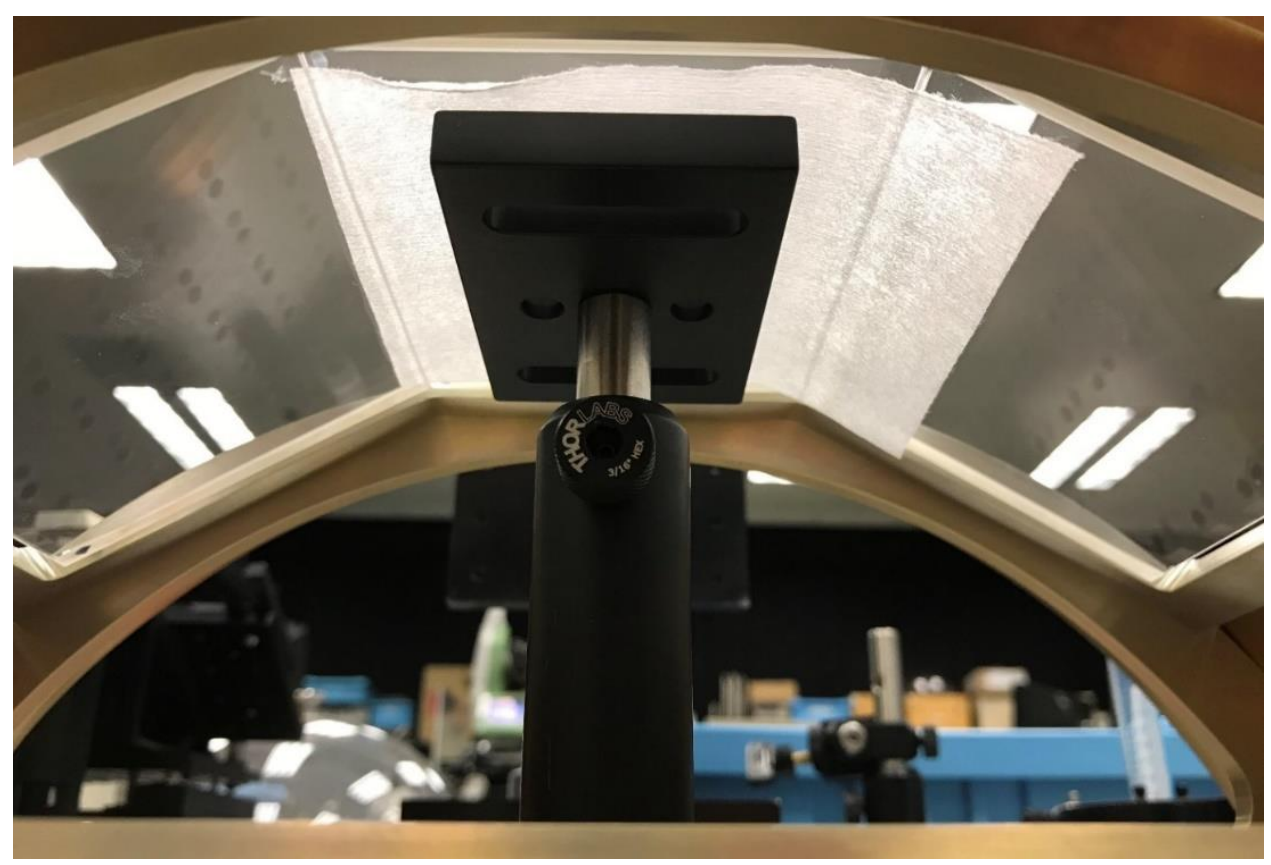

Figure 18. Window sub-assembly supported within frame from beneath

The underside of the bond line channels was sealed with 3M ESD tape, as shown in Figure 19. Dow Corning 6-1104, a low outgassing silicone adhesive, was injected into the taped channels from above. The adhesive was smoothed and allowed to cure overnight.

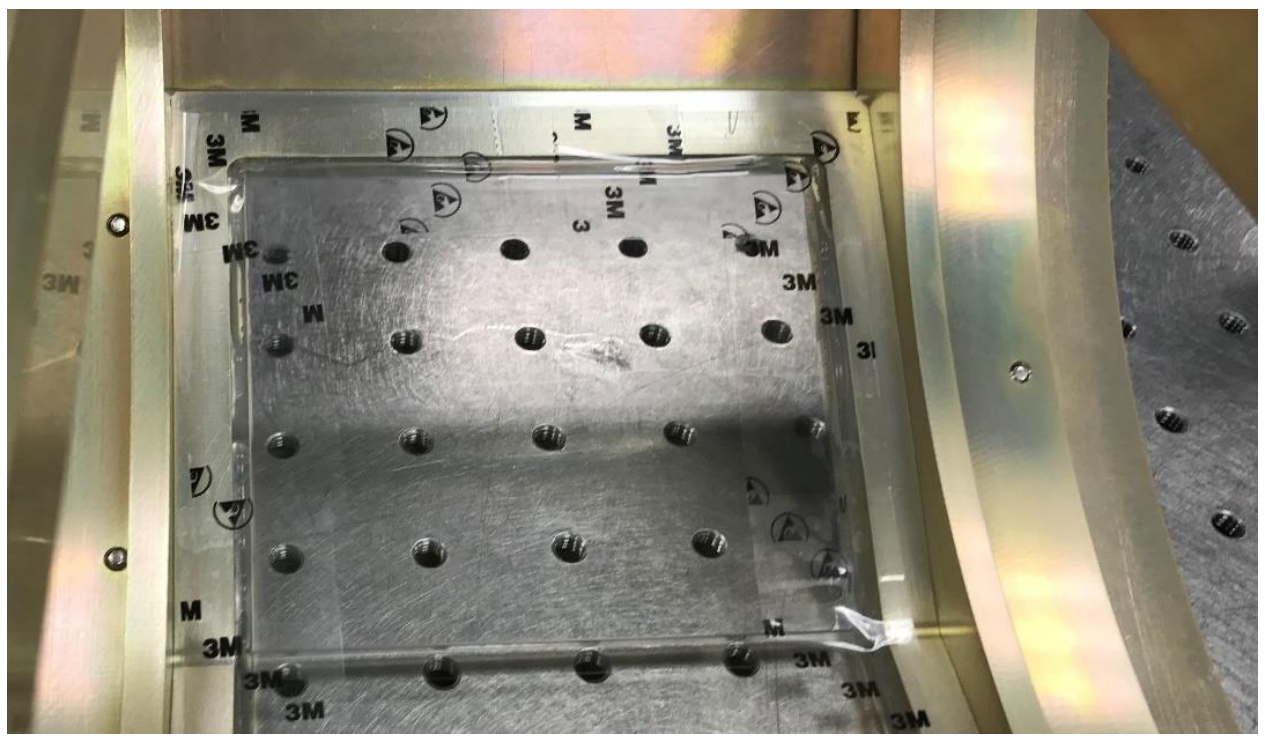

Figure 19. Taped bond line channels, from inside

The tape was removed from the underside. The bond was inspected for gaps and back-filled. Excess adhesive was mechanically removed from the frame and panes. The windowpanes were cleaned with lint-free wipes (Texwipes TX404) dampened in acetone first, then with isopropyl alcohol. The completed assembly is shown in Figure 20 through Figure 22. 


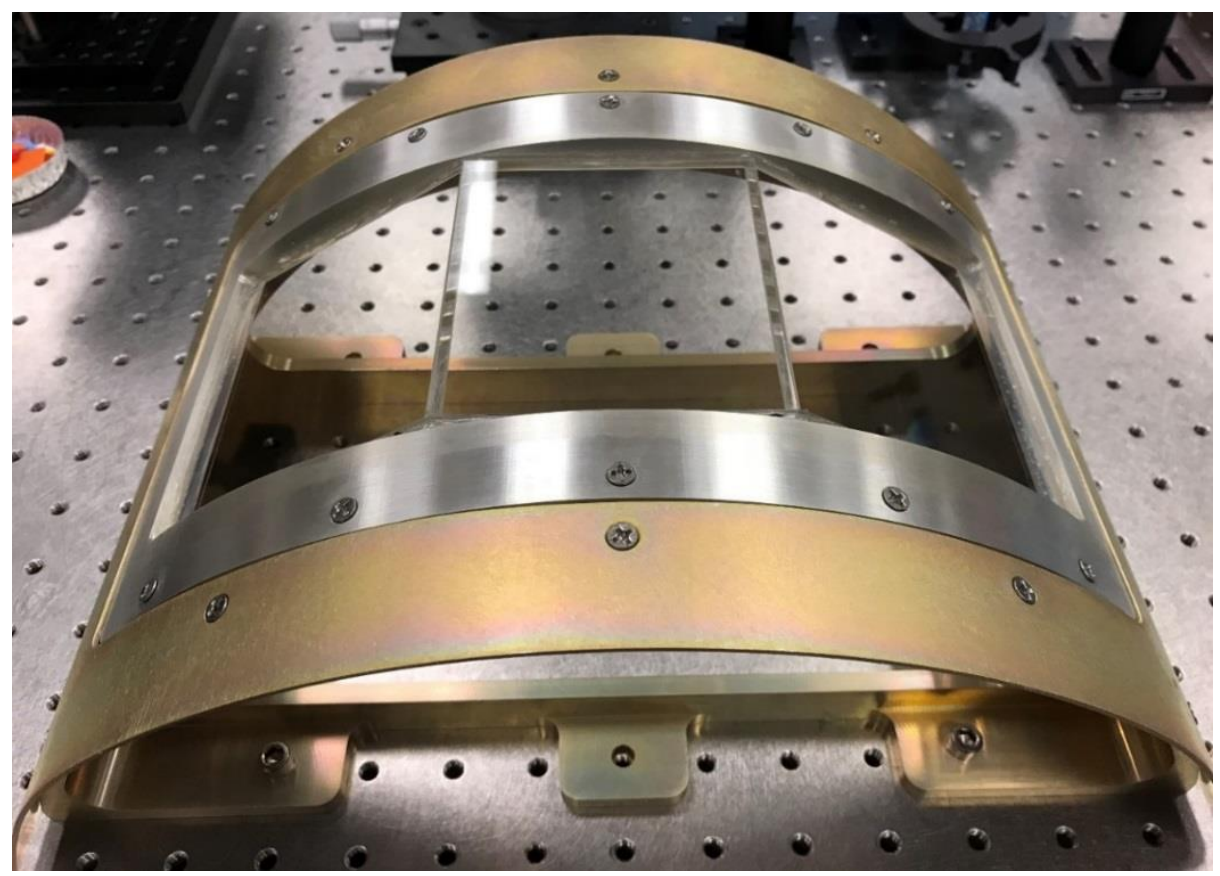

Figure 20. Completed near-conformal window assembly

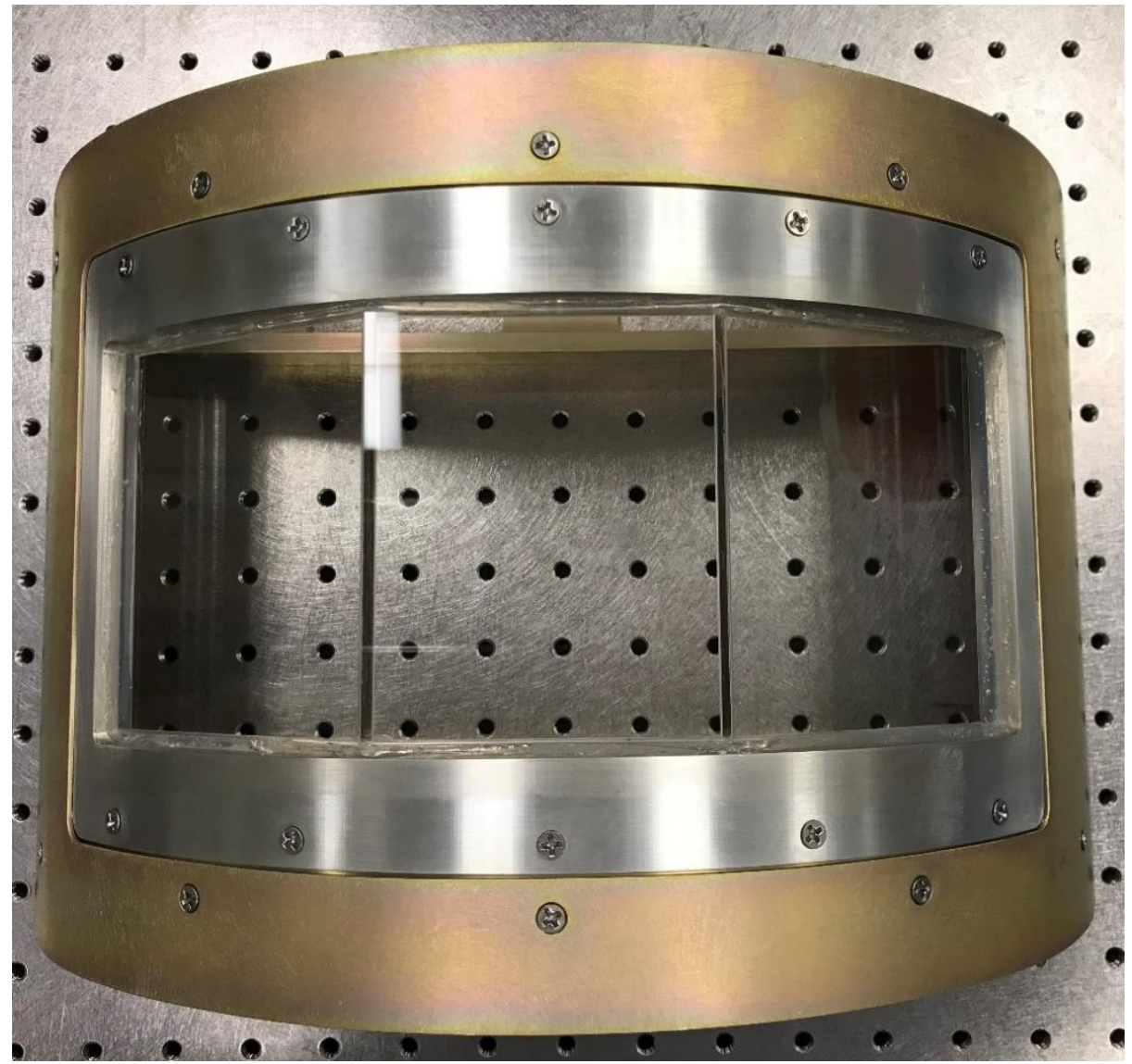

Figure 21. Near-conformal window assembly prototype, from outside 




Figure 22. Near-conformal window assembly prototype, from inside

The prototype's silicate bond lines were visually inspected, then it was placed in a thermal chamber. The chamber was cycled through five cycles from -40 to $+71^{\circ} \mathrm{C}$, with 1 hour dwells and a ramp rate of $1{ }^{\circ} \mathrm{C} /$ minute. After completion, the bond lines were re-inspected. There was no sign of bond line degradation.

\section{FUTURE TESTING/QUALIFICATION}

There are additional tests that would be helpful for characterizing the robustness and performance of the assembly. Environmental testing to survival levels for a notional platform would verify strength against representative aircraft loads. These tests could include random vibration ( 8 grms, 3 orthogonal axes), and shock (6 g, $11 \mathrm{~ms}$ half sine, 3 axes). Performance tests could include transmitted wavefront error over temperature, modulation contrast function (MTF), and even aero-optical characterization (e.g. pointing jitter in a wind tunnel).

\section{SUMMARY}

The near-conformal window concept uses multiple mitered windowpanes to mimic the local geometry of the aircraft or pod skin to reduce boundary layer turbulence. Reduced turbulence decreases aerodynamic drag and aero-optical effects, thereby improving aircraft time-on-station and optical payload performance. The near-conformal window approach provides a lower cost and shorter lead-time solution compared with monolithic windows that are ground and polished into complex geometries. The near-conformal window concept should be particularly useful for rapid prototyping of airborne optical payloads while the production windows are being manufactured.

The outcomes from this effort include:

- Establishment of a design and build process for near-conformal segmented window assemblies for aircraft optical payloads, in particular for silicate-bonded fused silica windowpanes

- Analytic prediction of aerodynamic performance benefit for a pod-mounted near-conformal window

- A hardware prototype that demonstrates the feasibility and utility of this approach. 


\section{ACKNOWLEDGEMENTS}

This material is based upon work supported by the United States Air Force under Air Force Contract No. FA8702-15-D0001. Any opinions, findings, conclusions or recommendations expressed in this material are those of the author(s) and do not necessarily reflect the views of the United States Air Force.

DISTRIBUTION STATEMENT A. Approved for public release. Distribution is unlimited.

\section{REFERENCES}

[1] General Atomics Aeronautical Systems Inc., "MQ-9 Reaper/Predator B Persistent Multi-Mission ISR," http://www.ga-asi.com/Websites/gaasi/images/products/aircraft_systems/pdf/MQ9\%20Reaper_Predator_B_032515.pdf (26 February 2018).

[2] Shepard, R.H., Rachlin, Y., Shah, V., Shih, T., "Design architectures for optically multiplexed imaging," Optics Express 23(24), 31419-31436 (2015).

[3] Hargraves, C., "IR sensor and window system issues," Proc. SPIE 1760, 331-334 (1992).

[4] Jones, M., "Optical analysis of segmented aircraft windows," Proc. SPIE 1498, 110-123 (1991).

[5] Hartmann, R., "Airborne FLIR optical window examples," Proc. SPIE 1760, 87, 90-93 (1992).

[6] Fisher, R.W., "Windows for standoff reconnaissance - a new approach,” Proc. SPIE 242, 57 (1980).

[7] Casteleiro, C., "Optical segmented RF signature managed window," US Patent 6560050 B2 (2003).

[8] Gentilman, R., "High strength edge-bonded sapphire windows," Proc. SPIE 3705, 282-287 (1999).

[9] Yoder, P.R., [Opto-Mechanical System Design], $3^{\text {rd }}$ ed., SPIE Press, Bellingham WA, 315-317 (2006).

[10] Shorey, A.B., Kordonski, W., Tracy, J., Tricard, M., "Developments in the finishing of domes and conformal optics," Proc. SPIE 6545, 65450Q-1 - 65450Q-9 (2007).

[11] Lockheed Martin Corp., "Electro-Optical Targeting System," mfc-f-35-eots-pc-001.pdf, https://www.lockheedmartin.com/en-us/products/f-35-lightning-ii-eots.html (19 July 2019).

[12] Lockheed Martin Corp., "Sniper Advanced Targeting Pod,"

https://www.lockheedmartin.com/content/dam/lockheed-martin/mfc/photo/sniper-pod/rotator/mfc-sniper-slider-imagefirst.jpg (12 August 2019).

[13] Armandula, H., "Silicate bonding procedure (hydroxide-catalysis bonding)," LIGO Laboratory, LIGO-E050228-v2 (2010). 